\title{
Resource Allocation Algorithm Based on Simultaneous Wireless Information and Power Transfer for OFDM Relay Networks
}

\author{
Zhenwei Xie ${ }^{1}$, Qi Zhu' ${ }^{1}$ and Su Zhao ${ }^{1,2}$ \\ ${ }^{1}$ The Key Wireless Laboratory of Jiangsu Province, School of Telecommunication and Information \\ Engineering, Nanjing University of Posts and Telecommunications \\ Nanjing 210003, Jiangsu - P. R. China \\ [e-mail: zhuqi@njupt.edu.cn] \\ ${ }^{2}$ National Mobile Communications Research Laboratory, Southeast University \\ Nanjing 210003, Jiangsu - P. R. China \\ *Corresponding author: Qi Zhu
}

Received February 15, 2017; revised July 1, 2017; accepted August 14, 2017; published December 31, 2017

\begin{abstract}
A resource allocation algorithm based on simultaneous wireless information and power transfer (SWIPT) to maximize the system throughput is proposed in orthogonal frequency division multiplexing (OFDM) relay networks. The algorithm formulates the problem under the peak power constraints of the source and each subcarrier (SC), and the energy causality constraint of the relay. With the given SC allocation of the source, we give and prove the optimal propositions of the formulated problem. Then, the formulated problem could be decomposed into two separate throughput maximization sub-problems by setting the total power to transfer energy. Finally, several SC allocation schemes are proposed, which are energy priority scheme, information priority scheme, balanced allocation scheme and exhaustive scheme. The simulation results reveal that the energy priority scheme can significantly reduce computational complexity and achieve approximate performance with the exhaustive scheme.
\end{abstract}

Keywords: decode-and-forward (DF) relay, OFDM, resource allocation, system throughput maximization, SWIPT

This research was supported by National Natural Science Foundation of China (61571234, 61631020), National Basic Research Program of China (973 program: 2013CB329005), the open research fund of National Mobile Communications Research Laboratory, Southeast University(No.2015D10). 


\section{Introduction}

SWIPT [1-2] can use the same radio wave to transfer information and energy to the receiver simultaneously, and the receiver can harvest energy via energy harvesting technology [3-4], which can greatly extend the lifecycle of the energy-constrained equipment, reduce charging cost and improve the performance of wireless communication systems.

There are many techniques that can be combined with SWIPT to improve network performance, such as cognitive radio technology, OFDM technology and relay technology, etc. In [5], a network selection and channel allocation mechanism to minimize accumulated interference and price is proposed. In [6], a new sufficient condition for the convergence of iterative waterfilling is studied. In [7], a random hypergraph based unified matching framework is proposed, under which five feasible types of multi-flow DF cooperative networks are considered. More importantly, the process of solving the problem give us great inspiration to use the related technology.

The resource allocation problems of energy harvesting wireless networks have been widely investigated. In [8], the throughput maximization problem is studied by the Lagrangian multiplier method and KKT conditions for a two-node energy harvesting wireless network, and a directional water-filling algorithm is obtained. In [9], the authors assume that battery capacity, data buffer size and data transmission delay of the source are limited, and the throughput maximization problem is decoupled into a power allocation problem and a data transmission problem. Then, the authors solve the original problem through an alternating maximization algorithm. In [10], under the constraints of data rate and energy harvest save ratios, the authors propose a novel best cooperative mechanism by maximizing the system throughput for energy harvesting and spectrum sharing in 5G networks. In [11], the power splitting (PS) and time switching (TS) protocols are taken into consideration, separately. Then, the expressions of the system outage probability under the two protocols are obtained. In [12], the authors propose a centralized algorithm to maximize the minimum signal. Analogously, the algorithm consideres the peak power constraints and the minimum harvested energy constraints of the receivers. In [13], the authors assume that the source uses the TS protocol to transfer information and energy, and the optimal TS coefficient problems are studied for three different transmission modes. In [14], the authors consider a practical assumption: Each transmitter only knows its own CSI to all the receivers. Then, an improved alternating direction method in a SWIPT multiple-input single-output system is proposed to maximize the total harvested energy of the receivers. But [11-14] only consider the use of the single-carrier SWIPT strategy, without considering the widely used OFDM technology.

In [15-16], the authors consider a point-to-point OFDM system with a DF relay. Then, a joint subcarrier pairing and power allocation algorithm to maximize the transmission rate of the system is proposed. In [17], the power allocation and subcarrier-relay assignment are 
jointly optimized to maximize the signal-to-noise ratio of the poorest channel. But in [15-17], all SCs are used to transfer information. When we combine OFDM technology with SWIPT technology and relay technology, the way to solve the problem will be very different.

OFDM technology can divide the spectrum into some orthogonal SCs, and each SC can transfer information or energy to the receiver. So OFDM technology is able to be combined with SWIPT technology (see, e.g., [18-26]). In [18-20], the broadcast single-cell networks by using the PS protocol are taken into consideration. In [18], the authors assume that energy can be transmitted in the downlink direction, and information can be transmitted in two direction. Several power-control algorithms are proposed to optimize both the throughput and energy transfer efficiency in different system configurations. In [19], the authors consider two cases where the receiver can harvest energy in a continuous or discrete mode, and a suboptimal resource allocation algorithm is obtained. In [20], the PS coefficient and SC allocation are simultaneously optimized by maximizing the energy harvested by all receivers, subject to the minimum rate constraint of each user. In [21], it is assumed that the receiver can work under the PS and TS protocols. Under the weighted sum-rate maximization criteria, the power allocation and SC allocation are jointly optimized by the Lagrangian multiplier method and an iterative algorithm. In [22], the authors propose a joint SC and power allocation SWIPT scheme in OFDM systems. In [23], it is assumed that the source can harvest energy from an energy supply point through some SCs, and transfer information to the destination through the remaining SCs. Then, the authors jointly optimize the SC allocation and power allocation by the variable substitution and Lagrangian multiplier method. But in [21-23], relay technology is not taken into consideration. In [24], the optimal TS and PS scheme is proposed for the two-hop SWIPT OFDM relay systems with the PS and TS protocols. In [25], the authors investigate the effects of the relay position and the number of SCs for the two-hop multiple-input multiple-output SWIPT OFDM relay systems with the PS and TS protocols, separately. In [26], the authors consider two cases where the direct link is available or unavailable for a two-hop SWIPT OFDM relay network. In this way, an algorithm to maximize the throughput is proposed by the ellipsoid method. But in [24-26], the power output capability of each SC is not taken into consideration.

The two-hop network model in this paper is shown in Fig. 1. In the 1st timeslot, the source transfers energy to the relay through some SCs and transfers information to the relay through the remaining SCs. In the 2nd timeslot, the relay can use all the SCs to transfer information to the destination. The proposed algorithm can flexibly adjust the number of the SCs used to transfer energy according to the channel condition, and then achieve the approximate performance of the exhaustive scheme with a lower computational complexity.

The main work of this paper is as follows:

1. It is assumed that the source can simultaneously transfer information and energy to the relay through different SCs respectively. Then, this paper formulates the throughput maximization problem under the peak power constraints of the source and each SC, and the 
energy causality constraint of the relay.

2. With the given SC allocation of the source, we give and prove the optimal propositions of the formulated problem. Proposition 1 gives the unequal relationship between the channel capacities of the source and relay. Proposition 2 shows the optimal strategy for the SCs to transfer energy. Proposition 3 shows that the maximum throughputs of the source and relay are monotone and continuous functions of the total power to transfer energy.

3. With the given SC allocation of the source, we decouple the system throughput maximization problem into two separate throughput maximization sub-problems by setting the total power to transfer energy from the source to the relay, and the two sub-problems can be solved by the water-filling method.

4. This paper proposes several simplified SC allocation schemes, which are energy priority scheme, information priority scheme and balanced allocation scheme.

This paper has the following structure. Section 2 gives the OFDM relay network model and throughput maximization problem. Section 3 develops an optimal algorithm with the given SC allocation. Section 4 proposes several simplified SC allocation schemes. Finally, Sections 5 and 6 give the simulation results and related conclusions.

\section{System Model and Problem Formulation}

We study the classic three-node OFDM relay network based on SWIPT, consisting of a source $\mathbf{S}$, a relay $\mathbf{R}$ and a destination $\mathbf{D}$, as shown in Fig. 1, where the $\mathbf{R}$ is an energy harvesting node with initial energy $E_{\mathbf{0}}$. The communication process is completed by two equal length timeslots. For convenience, the length of each timeslot is unitized to 1 , and the related conclusions can be extended to arbitrary timeslot length. At the same time, the system bandwidth $W$ is divided into $N$ equal orthogonal SCs, and the SC set is represented as $n \in \mathbf{N}=\{1, \ldots, N\}$. Assuming that each channel is a Rayleigh fading channel, and the channel gain of S-R at the SC $\boldsymbol{n}$ is $g_{n}$, and that of R-D at the SC $\boldsymbol{n}$ is $h_{n}$.

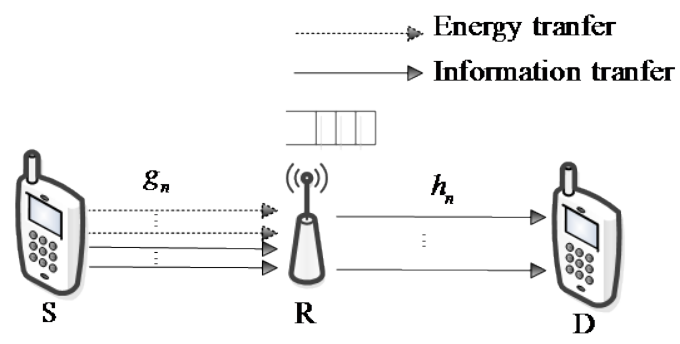

Fig. 1. OFDM relay network model based on SWIPT 
The source can simultaneously transfer information and energy to the relay through different SCs (see, e.g., [27-29]). The relay is assumed to adapt bandpass filters, which makes it possible to tap into different subcarriers [30]. Since the source does not need to transfer energy to the relay when $E_{0}$ is large enough, we define a SC $n=0$ with $g_{0}=0$ to mean that no SCs are used to transfer energy, and the set of all the SCs is denoted as $\widetilde{\mathbf{N}}=\{0\} \cup \mathbf{N}$. In the 1st timeslot, $\widetilde{\mathbf{N}}$ is divided into two complementary sets $\mathbf{N}^{\mathrm{E}}$ and $\mathbf{N}^{\mathbf{I}}$, which are respectively used to transfer energy and information, and the transmission power at the SC $n$ is $p_{n}, n \in \widetilde{\mathbf{N}}$. In the 2nd timeslot, the relay can transfer information through all the SCs, and the transmission power at the SC $n$ is $q_{n}, n \in \mathbf{N}$.

Supposing that the peak power constraint of the $\mathrm{S}$ is $Q$, so $p_{n}$ should satisfy

$$
\sum_{n \in \mathrm{N}^{\mathrm{E}}} p_{n}+\sum_{n \in \mathrm{N}^{\mathrm{I}}} p_{n} \leq Q
$$

The energy consumed at the relay cannot exceed the sum of the initial energy and harvested energy, i.e.,

$$
\sum_{n=1}^{N} q_{n} \leq \eta \sum_{n \in \mathbf{N}^{\mathrm{E}}} p_{n} g_{n}+E_{0}
$$

where $\eta, 0 \leq \eta \leq 1$ denotes the energy transfer efficiency.

The data transmission rate of the system must be less than or equal to the channel capacities of S-R and R-D, so as to ensure that the destination completely receives the data sent by the source, so:

$$
C \leq \min \left\{\sum_{n=1}^{N} \frac{1}{2} \log _{2}\left(1+\frac{q_{n} h_{n}}{\sigma_{D, n}^{2}}\right), \sum_{n \in \mathbf{N}^{\mathrm{I}}} \frac{1}{2} \log _{2}\left(1+\frac{p_{n} g_{n}}{\sigma_{R, n}^{2}}\right)\right\}
$$

where $\sigma_{R, n}^{2}$ and $\sigma_{D, n}^{2}$ denote the receiver noise power of the $\mathrm{R}$ and $\mathrm{D}$, respectively.

We can formulate the throughput maximization problem as:

$$
\begin{array}{ll}
\max _{\left\{p_{n}, q_{n}, \mathbf{N}^{\mathrm{E}}, \mathbf{N}^{\mathrm{I}}\right\}} & \min \left\{\sum_{n=1}^{N} \frac{1}{2} \log _{2}\left(1+\frac{q_{n} h_{n}}{\sigma_{D, n}^{2}}\right), \sum_{n \in \mathbf{N}^{\mathrm{I}}} \frac{1}{2} \log _{2}\left(1+\frac{p_{n} g_{n}}{\sigma_{R, n}^{2}}\right)\right\} \\
\text { s.t. } & \sum_{n \in \mathbf{N}^{\mathrm{E}}} p_{n}+\sum_{n \in \mathbf{N}^{\mathrm{I}}} p_{n} \leq Q \\
& \sum_{n=1}^{N} q_{n} \leq \eta \sum_{n \in \mathbf{N}^{\mathrm{E}}} p_{n} g_{n}+E_{0} \\
& p_{n} \leq P^{\max }, n \in \mathbf{N} \\
& q_{n} \leq P^{\max }, n \in \mathbf{N}
\end{array}
$$

where $P^{\max }$ represents the peak power constraint of each SC, and the source sends data at the maximum achievable rate $\min \left\{\sum_{n=1}^{N} \frac{1}{2} \log _{2}\left(1+\frac{q_{n} h_{n}}{\sigma_{D, n}^{2}}\right), \sum_{n \in \mathrm{N}^{1}} \frac{1}{2} \log _{2}\left(1+\frac{p_{n} g_{n}}{\sigma_{R, n}^{2}}\right)\right\}$. 


\section{Optimal Power Allocation with the Given SC Allocation}

First, two propositions are given for the optimal power allocation of problem (4). Proposition 1 gives the unequal relationship between the channel capacities of the source and relay. Proposition 2 shows the optimal strategy to transfer energy.

Proposition 1: There exists an optimal power allocation $\left\{p_{n}^{*}\right\}$ and $\left\{q_{n}^{*}\right\}$ of problem (4) satisfies

$$
\sum_{n \in \mathrm{N}^{\mathrm{I}}} \frac{1}{2} \log _{2}\left(1+\frac{p_{n}^{*} g_{n}}{\sigma_{R, n}^{2}}\right) \leq \sum_{n=1}^{N} \frac{1}{2} \log _{2}\left(1+\frac{q_{n}^{*} h_{n}}{\sigma_{D, n}^{2}}\right)
$$

Proof: Assuming that there exists an optimal power allocation $\left\{p_{n}^{*}\right\}$ and $\left\{q_{n}^{*}\right\}$ of problem (4), which doesn't satisfy (5), i.e., $\sum_{n \in \mathrm{N}^{\mathrm{N}}} \frac{1}{2} \log _{2}\left(1+\frac{p_{n}^{*} g_{n}}{\sigma_{R, n}^{2}}\right)>\sum_{n=1}^{N} \frac{1}{2} \log _{2}\left(1+\frac{q_{n}^{*} h_{n}}{\sigma_{D, n}^{2}}\right)$. Now, the optimal solution of problem (4) is $\min \left\{\sum_{n=1}^{N} \frac{1}{2} \log _{2}\left(1+\frac{q_{n}^{*} h_{n}}{\sigma_{D, n}^{2}}\right), \sum_{n \in \mathbf{N}^{\mathrm{N}}} \frac{1}{2} \log _{2}\left(1+\frac{p_{n}^{*} g_{n}}{\sigma_{R, n}^{2}}\right)\right\}=\sum_{n=1}^{N} \frac{1}{2} \log _{2}\left(1+\frac{q_{n}^{*} h_{n}}{\sigma_{D, n}^{2}}\right)$. Then, we can choose any one of $n_{1} \in \mathbf{N}^{\mathbf{I}}$, and reduce the power $p_{n_{1}}^{*}$ until $\sum_{n \in \mathbf{N}^{\mathbf{1}}} \frac{1}{2} \log _{2}\left(1+\frac{p_{n}^{*} g_{n}}{\sigma_{R, n}^{2}}\right)=\sum_{n=1}^{N} \frac{1}{2} \log _{2}\left(1+\frac{q_{n}^{*} h_{n}}{\sigma_{D, n}^{2}}\right)$ or $p_{n_{1}}^{*}=0$. If $\sum_{n \in \mathbb{N}^{1}} \frac{1}{2} \log _{2}\left(1+\frac{p_{n}^{*} g_{n}}{\sigma_{R, n}^{2}}\right)>\sum_{n=1}^{N} \frac{1}{2} \log _{2}\left(1+\frac{q_{n}^{*} h_{n}}{\sigma_{D, n}^{2}}\right)$ is still satisfied, we can choose any one of $n_{2} \in \mathbf{N}^{\mathrm{I}}, n_{2} \neq n_{1}$, and reduce the power $p_{n_{2}}^{*}$ until $\sum_{n \in \mathbf{N}^{\mathbf{1}}} \frac{1}{2} \log _{2}\left(1+\frac{p_{n}^{*} g_{n}}{\sigma_{R, n}^{2}}\right)=\sum_{n=1}^{N} \frac{1}{2} \log _{2}\left(1+\frac{q_{n}^{*} h_{n}}{\sigma_{D, n}^{2}}\right)$ or $p_{n_{2}}^{*}=0$. This process will be repeated until $\sum_{n \in \mathrm{N}^{1}} \frac{1}{2} \log _{2}\left(1+\frac{p_{n}^{*} g_{n}}{\sigma_{R, n}^{2}}\right)=\sum_{n=1}^{N} \frac{1}{2} \log _{2}\left(1+\frac{q_{n}^{*} h_{n}}{\sigma_{D, n}^{2}}\right)$. Now, the optimal solution of problem (4) is still $\min \left\{\sum_{n=1}^{N} \frac{1}{2} \log _{2}\left(1+\frac{q_{n}^{*} h_{n}}{\sigma_{D, n}^{2}}\right), \sum_{n \in \mathbf{N}^{\mathrm{N}}} \frac{1}{2} \log _{2}\left(1+\frac{p_{n}^{*} g_{n}}{\sigma_{R, n}^{2}}\right)\right\}=\sum_{n=1}^{N} \frac{1}{2} \log _{2}\left(1+\frac{q_{n}^{*} h_{n}}{\sigma_{D, n}^{2}}\right)$, because $\left\{q_{n}^{*}\right\}$ does not change. So, Proposition 1 is proved.

Proposition 2: In the optimal strategy, if $0<p_{\alpha(n)}^{*}<P^{\max }$ for $\forall n \in \mathbf{N}^{\mathbf{E}}$ with the given SC allocation $\mathbf{N}^{\mathrm{E}}$ and $\mathbf{N}^{\mathrm{I}}$, we can obtain

$$
p_{\alpha(m)}^{*}= \begin{cases}P^{\max }, & \text { if } 1 \leq m<n \\ 0, & \text { if } n<m \leq\left|\mathbf{N}^{\mathrm{E}}\right|\end{cases}
$$

where $\left|\mathbf{N}^{\mathbf{E}}\right|$ represents the number of the SCs contained in the set $\mathbf{N}^{\mathrm{E}}$. Vector $\boldsymbol{\alpha}$ represents the subscript of the channel gains sorted in descending order for $n \in \mathbf{N}^{\mathrm{E}}$, i.e., $\boldsymbol{\alpha}=\arg \operatorname{sort}\left(g_{n}\right), n \in \mathbf{N}^{\mathbf{E}}$, where the m-th element of $\boldsymbol{\alpha}$ is denoted as $\alpha(m)$.

The meaning of Proposition 2 is as follows. With the given SC allocation $\mathbf{N}^{\mathrm{E}}$ and $\mathbf{N}^{\mathbf{I}}$, 
we first select a SC which has the largest $g_{n}, n \in \mathbf{N}^{\mathrm{E}}$, i.e., $\alpha(1)$ to transfer energy. If the SC $\alpha(1)$ reaches the peak power constraint, then the second largest $g_{n}, n \in \mathbf{N}^{\mathrm{E}}$, i.e., $\alpha(2)$ is selected to transfer energy, and so on, until the relay harvests enough energy. The specific power allocation strategy is given in Subsection 3.1.

Proof: We will prove it by the reductio ad absurdum. In the optimal power allocation $\left\{p_{n}^{*}\right\}$ and $\left\{q_{n}^{*}\right\}$, assuming that $\exists u \in \mathbf{N}^{\mathrm{E}}$ does not satisfy (6). Now there may be two cases:

a) $\exists u \in[1, n)$ satisfies $0 \leq p_{\alpha(u)}^{*}<P^{\max }$.

b) $\exists u \in\left(n,\left|\mathbf{N}^{\mathrm{E}}\right|\right.$ ] satisfies $0<p_{\alpha(u)}^{*} \leq P^{\max }$.

For the case a), we can know $g_{\alpha(u)}>g_{\alpha(n)}$ from the definition of $\boldsymbol{\alpha}$, and let

$$
\begin{aligned}
& \bar{p}_{\alpha(n)}= \begin{cases}0 & , \text { if } p_{\alpha(u)}^{*}+p_{\alpha(n)}^{*} \leq P^{\max } \\
p_{\alpha(u)}^{*}+p_{\alpha(n)}^{*}-P^{\max }, & \text { if } p_{\alpha(u)}^{*}+p_{\alpha(n)}^{*}>P^{\max }\end{cases} \\
& \bar{p}_{\alpha(u)}= \begin{cases}p_{\alpha(u)}^{*}+p_{\alpha(n)}^{*}, & \text { if } p_{\alpha(u)}^{*}+p_{\alpha(n)}^{*} \leq P^{\max } \\
P^{\max } & \text {, if } p_{\alpha(u)}^{*}+p_{\alpha(n)}^{*}>P^{\max }\end{cases}
\end{aligned}
$$

At this time it is clear that all the constraints of problem (4) are still satisfied, and

$$
\bar{p}_{\alpha(u)} g_{\alpha(u)}+\bar{p}_{\alpha(n)} g_{\alpha(n)}>p_{\alpha(u)}^{*} g_{\alpha(u)}+p_{\alpha(n)}^{*} g_{\alpha(n)}
$$

From Proposition 1, we can obtain $\sum_{n \in \mathbf{N}^{\mathrm{I}}} \frac{1}{2} \log _{2}\left(1+\frac{p_{n}^{*} g_{n}}{\sigma_{R, n}^{2}}\right) \leq \sum_{n=1}^{N} \frac{1}{2} \log _{2}\left(1+\frac{q_{n}^{*} h_{n}}{\sigma_{D, n}^{2}}\right)$. Assuming that the allocated power of the other SCs will not change, and the relay can harvest more energy by (9). Let $\bar{p}_{\alpha(u)}=\bar{p}_{\alpha(u)}-\delta, \delta>0$, where $\delta$ must ensure that the inequality (9) holds. The source can have more power $\delta$ to transfer information, and the relay can have more power $\left(\bar{p}_{\alpha(u)}-\delta\right) g_{\alpha(u)}+\bar{p}_{\alpha(n)} g_{\alpha(n)}-p_{\alpha(u)}^{*} g_{\alpha(u)}-p_{\alpha(n)}^{*} g_{\alpha(n)}$ to transfer information. If $\exists n \in \mathbf{N}^{\mathrm{I}}, p_{n}^{*}<p_{n}^{\max }$ and $\exists n \in \mathbf{N}, q_{n}^{*}<P^{\max }$, the source and relay can achieve a higher system throughput than the strategy $\left\{p_{n}^{*}\right\}$ and $\left\{q_{n}^{*}\right\}$. Thus, the case a) does not hold.

It can be shown that the case b) does not hold in a similar manner to a). In summary, Proposition 2 has been demonstrated.

Supposing that the total power to transfer energy of the $\mathrm{S}$ is $Q_{1}$, and the total power to transfer information is $Q_{2}=Q-Q_{1}$. Then, $\sum_{n \in \mathbb{N}^{\mathrm{E}}} p_{n} \leq Q_{1}$ and $\sum_{n \in \mathrm{N}^{1}} p_{n} \leq Q_{2}$ must be satisfied. With the given $Q_{1}$ and SC allocation, the joint optimal power allocation strategy is as follows.

\subsection{Joint Optimal Power Allocation}

From Proposition 2, we first select the SC $\alpha(1)$ to transfer energy until $p_{\alpha(1)}=P^{\max }$ or $p_{\alpha(1)}=Q_{1}$. If $p_{\alpha(1)}<Q_{1}$, then we select the SC $\alpha(2)$ to transfer energy until $p_{\alpha(2)}=P^{\max }$ or 
$\sum_{n=1}^{2} p_{\alpha(n)}=Q_{1}$, and so on. This process will be repeated until $p_{\alpha(n)}=P^{\max }$ for $\forall n \in \mathbf{N}^{\mathrm{E}}$ or $\sum_{n=1}^{\left|\mathbf{N}^{\mathrm{E}}\right|} p_{\alpha(n)}=Q_{1}$. The specific solution of $Q_{1}$ is given in Subsection 3.2.

Now, the total energy of the relay $\eta \sum_{n \in \mathbf{N}^{\mathrm{E}}} p_{n} g_{n}+E_{0}$, and $Q_{2}=Q-Q_{1}$ can be obtained. So the feasible region of problem (4) for $p_{n}, n \in \mathbf{N}^{\mathbf{I}}$ and $q_{n}, n \in \mathbf{N}$ is separable, and problem (4) could be decomposed into the following two sub-problems:

$$
\begin{array}{cc}
\max _{\left\{p_{n}\right\}} & \sum_{n \in \mathbf{N}^{\mathrm{I}}} \frac{1}{2} \log _{2}\left(1+\frac{p_{n} g_{n}}{\sigma_{R, n}^{2}}\right) \\
\text { s.t. } & \sum_{n \in \mathbf{N}^{\mathrm{I}}} p_{n} \leq Q_{2} \\
& p_{n} \leq P^{\max }, n \in \mathbf{N}^{\mathbf{I}} \\
\max _{\left\{q_{n}\right\}} & \sum_{n=1}^{N} \frac{1}{2} \log _{2}\left(1+\frac{q_{n} h_{n}}{\sigma_{D, n}^{2}}\right) \\
\text { s.t. } & \sum_{n=1}^{N} q_{n} \leq \eta \sum_{n \in \mathbf{N}^{\mathrm{E}}} p_{n} g_{n}+E_{0}
\end{array}
$$

The Lagrangian function of problem (10) is

$$
\mathcal{L}=\sum_{n \in \mathbf{N}^{\mathbf{I}}} \frac{1}{2} \log _{2}\left(1+\frac{p_{n} g_{n}}{\sigma_{R, n}^{2}}\right)-\lambda\left(\sum_{n \in \mathbf{N}^{1}} p_{n}-Q_{2}\right)-\sum_{n \in \mathbf{N}^{1}} \mu_{n}\left(p_{n}-P^{\max }\right)
$$

where the Lagrange multipliers $\lambda$ and $\left\{\mu_{n}\right\}$ correspond to the two constraints in (10), respectively.

We apply the KKT conditions and partial derivative of $p_{n}$ to get

$$
p_{n}^{*}=\left(\frac{1}{2\left(\lambda+\mu_{n}\right) \ln 2}-\frac{\sigma_{R, n}^{2}}{g_{n}}\right)^{+}, n \in \mathbf{N}^{\mathbf{I}}
$$

The complementary slackness condition about $p_{n} \leq P^{\max }, n \in \mathbf{N}^{\mathbf{I}}$ is

$$
\mu_{n}\left(p_{n}-P^{\max }\right)=0
$$

We can get $\mu_{n}=0$ whenever $p_{n}<P^{\max }$ by (14). If $\mu_{n}>0$, we must have $p_{n}=P^{\max }$, i.e., $\mu_{n}$ forces $p_{n}$ to reduce until $p_{n}=P^{\max }$.

So the optimal solution of problem (10) is

$$
p_{n}^{*}=\min \left(\left(\frac{1}{2 \lambda \ln 2}-\frac{\sigma_{R, n}^{2}}{g_{n}}\right)^{+}, P^{\max }\right), n \in \mathbf{N}^{\mathbf{I}}
$$

where $(x)^{+}=\max (0, x)$, and $\lambda$ satisfies $\sum_{n \in \mathbf{N}^{\mathrm{I}}} p_{n}=Q_{2}$ or $p_{n}=P^{\max }$ for $\forall n \in \mathbf{N}^{\mathbf{I}}$.

Similarly, the optimal solution of problem (11) is 


$$
q_{n}^{*}=\min \left(\left(\frac{1}{2 \gamma \ln 2}-\frac{\sigma_{D, n}^{2}}{h_{n}}\right)^{+}, P^{\max }\right), n \in \mathbf{N}
$$

where $\gamma$ satisfies $\sum_{n=1}^{N} q_{n}=\eta \sum_{n \in \mathbf{N}^{\mathrm{E}}} p_{n} g_{n}+E_{0}$ or $q_{n}=P^{\max }$ for $\forall n \in \mathbf{N}$.

\subsection{Optimal Solution of $Q_{1}$}

We consider two cases where the initial energy of the relay satisfies $E_{0}=0$ or $E_{0}>0$. First, we give a proposition about how the maximum throughputs of the source and relay change with $Q_{1}$.

Proposition 3: With the continuous increase of $Q_{1}$, if $\exists n \in \mathbf{N}^{\mathrm{I}}, p_{n}^{*}<P^{\max }$, the maximum throughput of the source $R_{\mathrm{S}}^{*}=\sum_{n \in \mathrm{N}^{\mathrm{N}}} \frac{1}{2} \log _{2}\left(1+\frac{p_{n}^{*} g_{n}}{\sigma_{R, n}^{2}}\right)$ is a continuous and monotonically decreasing function; if $\exists n \in \mathbf{N}^{\mathbf{E}}, \quad p_{n}^{*}<p_{n}^{\max }$ and $\exists n \in \mathbf{N}, \quad q_{n}^{*}<P^{\max }$, the maximum throughput of the relay $R_{\mathrm{R}}^{*}=\sum_{n=1}^{N} \frac{1}{2} \log _{2}\left(1+\frac{q_{n}^{*} h_{n}}{\sigma_{D, n}^{2}}\right)$ is a continuous and monotonically increasing function.

Proof: First, we prove the monotonicity.

With the continuous increase of $Q_{1}, Q_{2}=Q-Q_{1}$ is continuously decreasing. Then, if $\exists n \in \mathbf{N}^{\mathrm{I}}, p_{n}^{*}<P^{\max }, \lambda$ must increase to accommodate the decrease of $Q_{2}$ by (15). So $R_{\mathrm{S}}^{*}$ is monotonically decreasing with the increase of $Q_{1}$.

For the relay, we can obtain at least the maximum throughput before the increase of $Q_{1}$. With the continuous increase of $Q_{1}$, if $\exists n \in \mathbf{N}^{\mathrm{E}}, p_{n}^{*}<p_{n}^{\max }$, the relay can harvest more energy. If $\exists n \in \mathbf{N}, q_{n}^{*}<P^{\max }, \gamma$ must decrease to accommodate the increase of $\eta \sum_{n \in \mathrm{N}^{\mathrm{E}}} p_{n} g_{n}+E_{0}$ by (16). So $R_{\mathrm{R}}^{*}$ is monotonically increasing with the decrease of $Q_{1}$.

Second, we prove the continuity.

Since the data that can be transmitted by limited energy in a limited time is also limited, the maximum throughputs of the source and relay will not jump. Meanwhile, the range of $Q_{1}$ is $0 \leq Q_{1} \leq Q$. That is, the continuity is guaranteed.

Similarly, we can get the extension of Proposition 3. With the continuous decrease of $Q_{1}$, if $\exists n \in \mathbf{N}^{\mathrm{I}}, p_{n}^{*}<p_{n}^{\max }, R_{\mathrm{S}}^{*}$ is continuous and monotonically increasing; if $\exists n \in \mathbf{N}$, $q_{n}^{*}<P^{\max }$ and $\exists n \in \mathbf{N}^{\mathrm{E}}, p_{n}^{*}<p_{n}^{\max }$, the maximum throughput of the relay $R_{\mathrm{R}}^{*}$ is continuous and monotonically decreasing.

When $E_{0}=0$, several typical situations about how $R_{\mathrm{S}}^{*}$ and $R_{\mathrm{R}}^{*}$ change with $Q_{1}$ are 
shown in Fig. 2, where the horizontal straight line of $R_{\mathrm{S}}^{*}$ is due to $p_{n}^{*}=P^{\max }$ for $\forall n \in \mathbf{N}^{\mathrm{I}}$, and the horizontal straight line of $R_{\mathrm{R}}^{*}$ is due to $q_{n}^{*}=P^{\max }$ for $\forall n \in \mathbf{N}$ or $p_{n}^{*}=P^{\max }$ for $\forall n \in \mathbf{N}^{\mathrm{E}}$. When we set different values of $P^{\max }$, there exist the following three typical cases, where the system maximum throughput curve is expressed as the smaller of $R_{\mathrm{S}}^{*}$ and $R_{\mathrm{R}}^{*}$ curves under the same $Q_{1}$, i.e., $\min \left(R_{S}^{*}, R_{R}^{*}\right)$, meanwhile, we ignore some insignificant situations. The system maximum throughput curve is shown in Fig. 2(a), and is omitted in Fig. 2(b) and Fig. 2(c). With the increase of $Q_{1}$ from zero to $Q$, the system maximum throughput must first increase, and then reach the maximum value, and then reduce to zero. Therefore, we could use some methods such as golden section method [31] to simplify the searching process of $Q_{1}$.

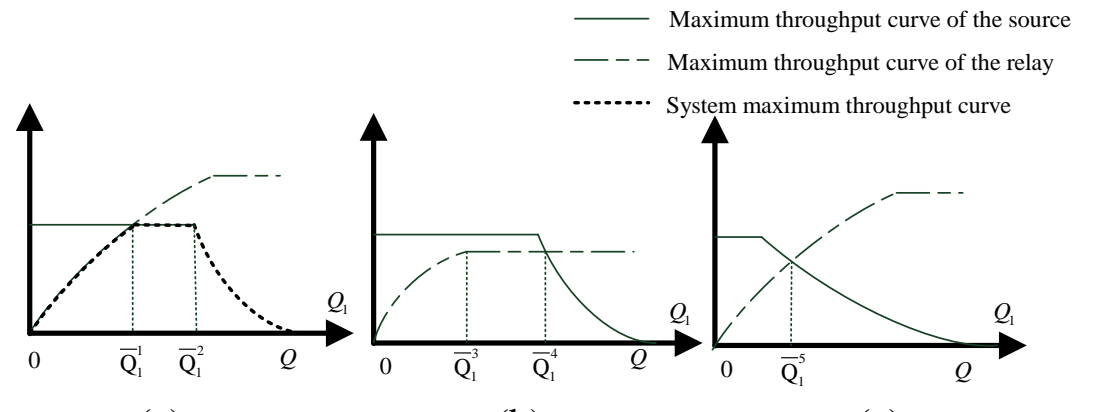

(a)

(b)

(c)

Fig. 2. Schematic diagram of the derivation process

When the initial energy of the relay $E_{0}>0$, there may exist a case where $E_{0}$ is very large, and even if $Q_{1}=0$, the maximum throughput of the source is still less than the relay's. Now the golden section method is no longer applicable, and we can use the following search method.

When $R_{\mathrm{S}}^{*}>R_{\mathrm{R}}^{*}$, if $\exists n \in \mathbf{N}^{\mathrm{E}}, p_{n}^{*}<p_{n}^{\max }$ and $\exists n \in \mathbf{N}, q_{n}^{*}<P^{\max }$, we can increase $Q_{1}$ until $p_{n}^{*}<P^{\max }$ for $\forall n \in \mathbf{N}^{\mathrm{E}}$ or $q_{n}^{*}=P^{\max }$ for $\forall n \in \mathbf{N}$ or $R_{\mathrm{S}}^{*}=R_{\mathrm{R}}^{*}$. From Proposition 3, with the increase of $Q_{1}, R_{\mathrm{R}}^{*}$ and the system maximum throughput $\min \left(R_{S}^{*}, R_{R}^{*}\right)$ both will increase, until the optimal $Q_{1}^{*}$ is obtained.

When $R_{\mathrm{S}}^{*}<R_{\mathrm{R}}^{*}$, if $\exists n \in \mathbf{N}^{\mathrm{I}}, p_{n}^{*}<P^{\max }$, we can decrease $Q_{1}$ util $Q_{1}=0$ or $p_{n}^{*}=P^{\max }$ for $\forall n \in \mathbf{N}^{\mathrm{I}}$ or $R_{\mathrm{S}}^{*}=R_{\mathrm{R}}^{*}$. With the continuous decrease of $Q_{1}, R_{S}^{*}$ and the system maximum throughput $\min \left(R_{S}^{*}, R_{R}^{*}\right)$ both will increase, until the optimal $Q_{1}^{*}$ is obtained.

From Proposition 3, $R_{\mathrm{S}}^{*}$ is non-increasing and $R_{\mathrm{R}}^{*}$ is non-decreasing with the increase of $Q_{1}$. Therefore, with the given SC allocation $\mathbf{N}^{\mathrm{E}}$ and $\mathbf{N}^{\mathrm{I}}$, the optimal solution of problem 
(4) must be unique and can be searched out by the above method. Note that $Q_{1}$ corresponding to the optimal value is not necessarily unique, as shown in Fig. 2(a) $\overline{\mathrm{Q}}_{1}^{1} \leq \mathrm{Q}_{1}^{*} \leq \overline{\mathrm{Q}}_{1}^{2}$ and Fig. 2(b) $\overline{\mathrm{Q}}_{1}^{3} \leq \mathrm{Q}_{1}^{*} \leq \overline{\mathrm{Q}}_{1}^{4}$.

The detailed optimal power allocation strategy is shown in Algorithm 1, where the range $a \leq x \leq b$ is denoted as $[a, b]$. The computational complexity of Algorithm 1 depends on the size of $s$. When we set a large $s$, the system throughput will converge quickly, but the throughput performance obtained will be poor because of poor calculation accuracy. On the contrary, when we set a small $s$, the system throughput will converge slowly, but the throughput performance obtained will be great because of high calculation accuracy.

Algorithm 1. Optimal Power Allocation with the Given SC Allocation

Input: range of $Q_{1},[0, Q]$; initial energy of the relay $E_{0}$; SC allocation $\mathbf{N}^{\mathrm{E}}$ and

$\mathbf{N}^{\mathrm{I}}$; number of the SCs $N$; channel gains $\left\{h_{n}\right\}$ and $\left\{g_{n}\right\}$

Output: system maximum throughput $C^{*}$; optimal power allocation $\left\{p_{n}^{*}\right\}$ and $\left\{q_{n}^{*}\right\}$

1. Initialize $Q_{1}=Q / 2, Q_{2}=Q-Q_{1}$, minimum step size $s$ of $Q_{1}$;

2. Obtain $p_{n}^{*}, n \in \mathbf{N}^{\mathrm{E}}$ from Proposition 2;

3. Solve $p_{n}^{*}, n \in \mathbf{N}^{\mathbf{I}}$ and $q_{n}^{*}, n \in \mathbf{N}$ by (15)(16);

4. Compute $R_{\mathrm{S}}^{*}=\sum_{n \in \mathrm{N}^{\mathrm{N}}} \frac{1}{2} \log _{2}\left(1+\frac{p_{n}^{*} g_{n}}{\sigma_{R, n}^{2}}\right)$ and $R_{\mathrm{R}}^{*}=\sum_{n=1}^{N} \frac{1}{2} \log _{2}\left(1+\frac{q_{n}^{*} h_{n}}{\sigma_{D, n}^{2}}\right)$;

5. if $R_{\mathrm{S}}^{*}<R_{\mathrm{R}}^{*}$

6. Repeat $Q_{1}=Q_{1}-s$ and steps 2,3 and 4 , until $Q_{1}=0$ or $p_{n}^{*}=P^{\max }$ for $\forall n \in \mathbf{N}^{\mathbf{I}}$ or $R_{\mathrm{S}}^{*}=R_{\mathrm{R}}^{*}$;

7. else

8. Repeat $Q_{1}=Q_{1}+s$ and steps 2,3 and 4, until $q_{n}^{*}=P^{\max }$ for $\forall n \in \mathbf{N}$ or $p_{n}^{*}<P^{\max }$ for $\forall n \in \mathbf{N}^{\mathrm{E}}$ or $R_{\mathrm{S}}^{*}=R_{\mathrm{R}}^{*}$;

9. end if

10. Compute $C^{*}=\min \left(R_{S}^{*}, R_{R}^{*}\right)$;

\section{SC Allocation Strategy}

Here, we consider the SC allocation of problem (4). Since the SC allocation of problem (4) is a integer programming problem, the computational complexity of the exhaustive scheme is $\mathcal{O}\left(2^{N}\right)$. So this paper proposes several simplified SC allocation schemes.

1. Energy priority scheme: First, all the SCs of the source are used to transfer 
information, i.e., $\mathbf{N}_{\mathbf{0}}^{\mathrm{I}}=\{1,2, \ldots, N\}, \mathbf{N}_{\mathbf{0}}^{\mathrm{E}}=\{0\}$. The relay uses the initial energy $E_{0}$ to transfer information, and then the system maximum throughput $C_{0}^{*}$ is calculated by Algorithm 1. This is because, when $E_{0}$ is very large, the system performance will obviously decline if some SCs are used to transfer energy. Then, the channel gains of the SCs $n \in \mathbf{N}$ at the source are sorted in descending order to obtain the subscript vector $\boldsymbol{\beta}$ at the original position, i.e., $\boldsymbol{\beta}=\arg \operatorname{sort}\left(g_{n}\right), n \in \mathbf{N}$. Afterward, we select the first $m(1 \leq m<|\mathbf{N}|)$ SCs of the vector $\boldsymbol{\beta}$ to transfer energy, i.e., $\mathbf{N}_{\mathbf{m}}^{\mathrm{E}}=\{\beta(1), \beta(2), \ldots, \beta(m)\}$, and the remaining SCs are used to transfer information. Meanwhile, the system maximum throughput $C_{m}^{*}$ is calculated by Algorithm 1. Finally, the system maximum throughput obtained by this scheme is $C^{*}=\max \left\{C_{m}^{*}\right\}, 0 \leq m<|\mathbf{N}|$, and the computational complexity of this scheme is $\mathcal{O}(N)$.

2. Information priority scheme: First, $C_{0}^{*}$ and $\boldsymbol{\beta}$ are obtained according to the above method in the energy priority scheme. Then, we select the first $m(1 \leq m<|\mathbf{N}|)$ SCs of the vector $\boldsymbol{\beta}$ to transfer information, i.e., $\mathbf{N}_{\mathrm{m}}^{\mathrm{I}}=\{\beta(1), \beta(2), \ldots, \beta(m)\}$, and the remaining SCs are used to transfer energy. Meanwhile, the system maximum throughput $C_{m}^{*}$ is calculated by Algorithm 1. Finally, $C^{*}=\max \left\{C_{m}^{*}\right\}, 0 \leq m<|\mathbf{N}|$, and the computational complexity of this scheme is $\mathcal{O}(N)$.

3. Balanced allocation scheme: First, $C_{0}^{*}$ and $\boldsymbol{\beta}$ are obtained, too. Then, there is a $50 \%$ probability for the SCs located in the odd locations of $\boldsymbol{\beta}$ to transfer information or energy, and the remaining SCs are used to transfer energy or information. Afterward, the system maximum throughput $C_{1}^{*}$ is calculated by Algorithm 1. Finally, $C^{*}=\max \left\{C_{0}^{*}, C_{1}^{*}\right\}$, and the computational complexity of this scheme is $\mathcal{O}(2)$.

In this paper, we use the exhaustive scheme to obtain the optimal SC allocation, namely, the performance upper bound of problem (4). In the exhaustive scheme, all the SCs of the source have two options, i.e., energy transmission and information transmission. So the computational complexity of the exhaustive scheme is $\mathcal{O}\left(2^{N}\right)$. On the other hand, due to the Section 3 gives the optimal power allocation with the given SC allocation. Then, we consider each SC allocation situation, and get the maximum throughput for each SC allocation situation. In this way, the maximum value of these maximum throughputs is the optimal solution of problem (4).

\section{Simulation and Analysis}

In this paper, the simulation parameters are set according to [26]. Assuming that the distance of S-D is $6 \mathrm{~m}$, and the relay is randomly distributed in a circle with a radius of $2 \mathrm{~m}$ centered on the mid-point of the $S$ and $D$. The initial energy of the relay is $E_{0}=0$, and the 
duration of each timeslot is $1 \mathrm{~s}$. The system bandwidth is $W=1 \mathrm{MHz}$, and the bandwidth of each SC is $\mathrm{W} / \mathrm{N}$. Assuming that the system coherence bandwidth is much larger than the bandwidth of each SC. Meanwhile, the large-scale fading is $\left(-31.5-30 \log _{10} d\right) \mathrm{dB}$ [32] at distance $d$, and the small-scale fading is Rayleigh fading. The noise power spectral density of the receivers is $N_{0}=-174 \mathrm{dBm} / \mathrm{Hz}$. The energy transfer efficiency is $\eta=0.2$.

We compare the maximum throughputs of the four SC allocation schemes by using Algorithm 1, which are called as energy priority algorithm, information priority algorithm, balanced allocation algorithm and exhaustive algorithm, respectively. Meanwhile, an equal power allocation (EPA) algorithm is compared, and it uses the exhaustive SC allocation scheme.

Fig. 3 shows the system maximum throughput comparison of the energy priority algorithm under different $s$, where $s$ represents the minimum step size of steps 6 and 8 in Algorithm 1. We set the peak power constraint of the source $Q=26 \mathrm{dBm}$, the number of the SCs $N=16$ and the peak power of each SC $P^{\max }=17 \mathrm{dBm}$. As can be seen from Fig. 3, the throughputs of all curves will converge to different constant values, and the final system throughput is larger for the curve with a smaller $s$. On the other hand, the average number of iterations of steps 6 and 8 in Algorithm 1 is more for the curve with a smaller $s$. That is to say, the computational complexity of Algorithm 1 depends on the size of $s$, and the system throughput performance with a smaller $s$ is better, but the computational complexity is higher.

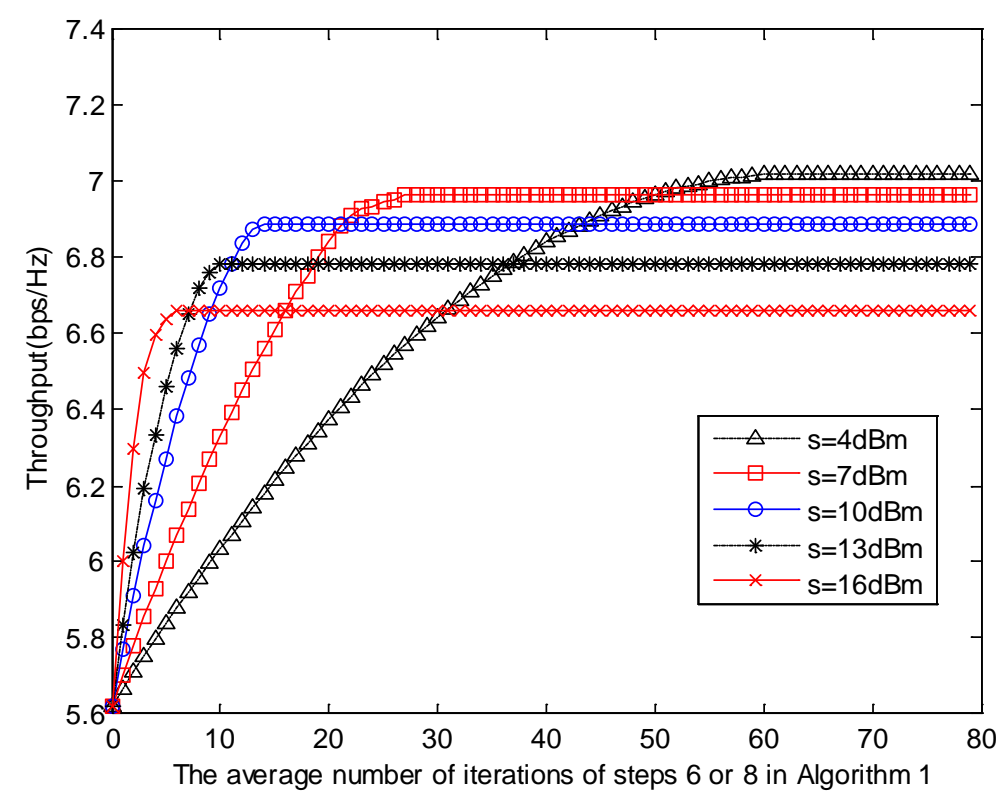

Fig. 3. System maximum throughput comparison of the energy priority algorithm under different $s$, where $Q=26 \mathrm{dBm}, N=16$ 
Fig. 4 shows the system maximum throughput comparison of different algorithms under different peak power constraints $Q$, where the number of the SCs is $N=16$ and the peak power of each SC is $P^{\max }=17 \mathrm{dBm}$. In Fig. 4, the energy priority algorithm achieves almost the same throughput as the exhaustive algorithm, and is better than the other three algorithms. The balanced allocation algorithm has a larger throughput than the information priority algorithm, because it has half the probability to use the SCs with larger channel gain to transfer energy. With the increase of $Q$, the throughput difference between the EPA algorithm and exhaustive algorithm will gradually reduce, because the probability that the allocated power of each SC reaches the peak power constraint is increasing.

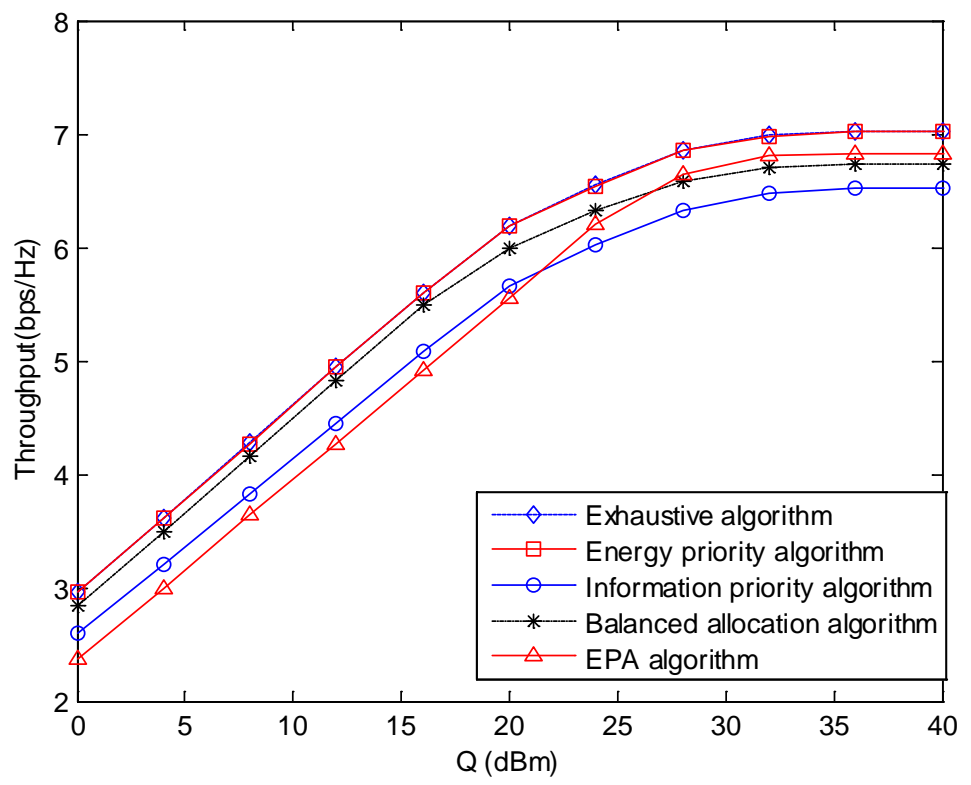

Fig. 4. System maximum throughput comparison of different algorithms under different $Q$, where

$$
N=16, P^{\max }=17 \mathrm{dBm}
$$

Fig. 5 shows the system maximum throughput comparison of different algorithms with no SC peak power constraint, where $N=16$. With the increase of $Q$, the energy priority algorithm and exhaustive algorithm are better than the other three algorithms. Meanwhile, the throughput difference between the balanced allocation algorithm and information priority algorithm becomes smaller and smaller, because the former uses a fixed number of SCs to transfer energy, while the latter can dynamically adjust the number of the SCs used to transfer energy. 


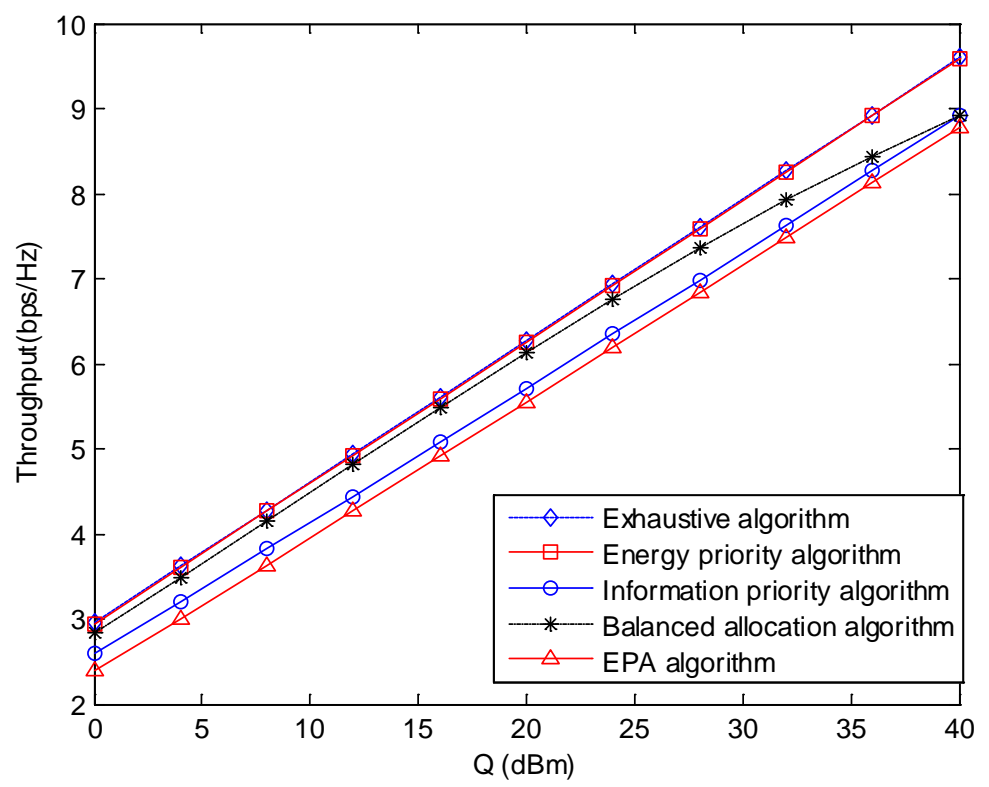

Fig. 5. System maximum throughput comparison of different algorithms with no SC peak power constraint, where $N=16$

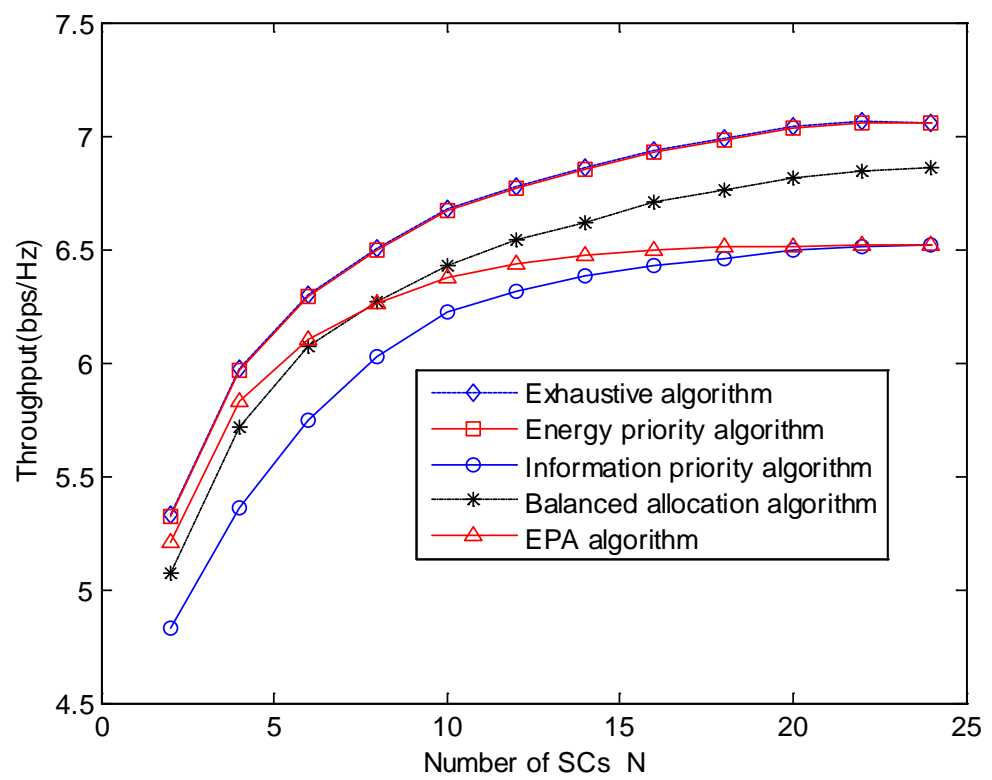

Fig. 6. System maximum throughput comparison of different algorithms under different $N$, where $Q=26 \mathrm{dBm}, P^{\max }=17 \mathrm{dBm}$ 
Fig. 6 shows the system maximum throughput comparison of different algorithms under different $N$, where $Q=26 \mathrm{dBm}$ and $P^{\max }=17 \mathrm{dBm}$. The energy priority algorithm achieves almost the same throughput as the exhaustive algorithm, and is better than the other algorithms, too. The balanced allocation algorithm has a larger throughput than the information priority algorithm. We observe that when the number of the SCs $N$ is small, the EPA algorithm has some advantages because it uses the exhaustive SC allocation scheme. Finally, the throughputs of different algorithms will converge due to the peak power constraint of the source $Q=26 \mathrm{dBm}$.

Fig. 7 shows the system maximum throughput comparison of the energy priority algorithm under different $Q$, where $N=16$. When $Q$ is small, the system throughput performance of different $P^{\max }$ is close. With the increase of $Q$, the system throughput performance with a larger $P^{\max }$ is better.

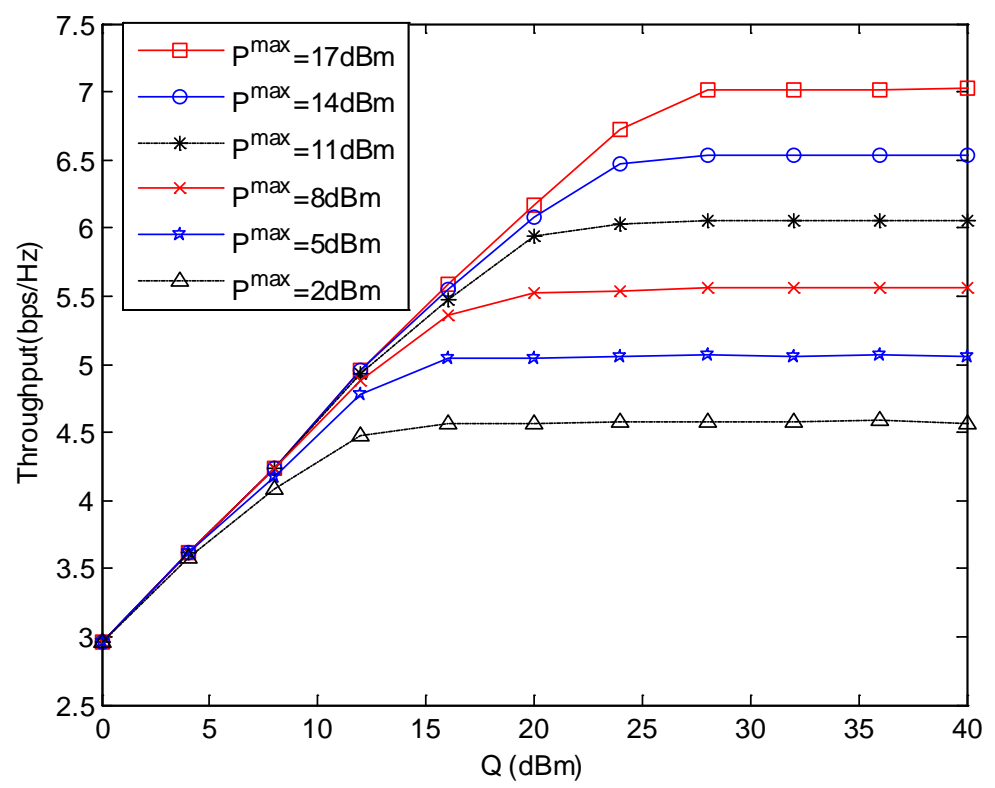

Fig. 7. System maximum throughput comparison of the energy priority algorithm under different $P^{\max }$, where $N=16$

Fig. 8 shows the average running time comparison of different algorithms under different number of the SCs $N$, where the peak power constraint of the source $Q=26 \mathrm{dBm}$, the minimum step size $s=4 \mathrm{dBm}$ and the peak power of each $\mathrm{SC}$ is $P^{\max }=17 \mathrm{dBm}$. Simulation computer is ASUS FX50JK4710 with 8G memory. The operating system is WIN10, and the version of MATLAB is 2014a. In Fig. 8, the energy priority algorithm and information priority algorithm have the same time complexity, which is far less than the time complexity of the exhaustive algorithm, especially when the number of SCs is large. 
The balanced allocation algorithm has some advantages over time complexity, and the EPA algorithm has the lowest time complexity.

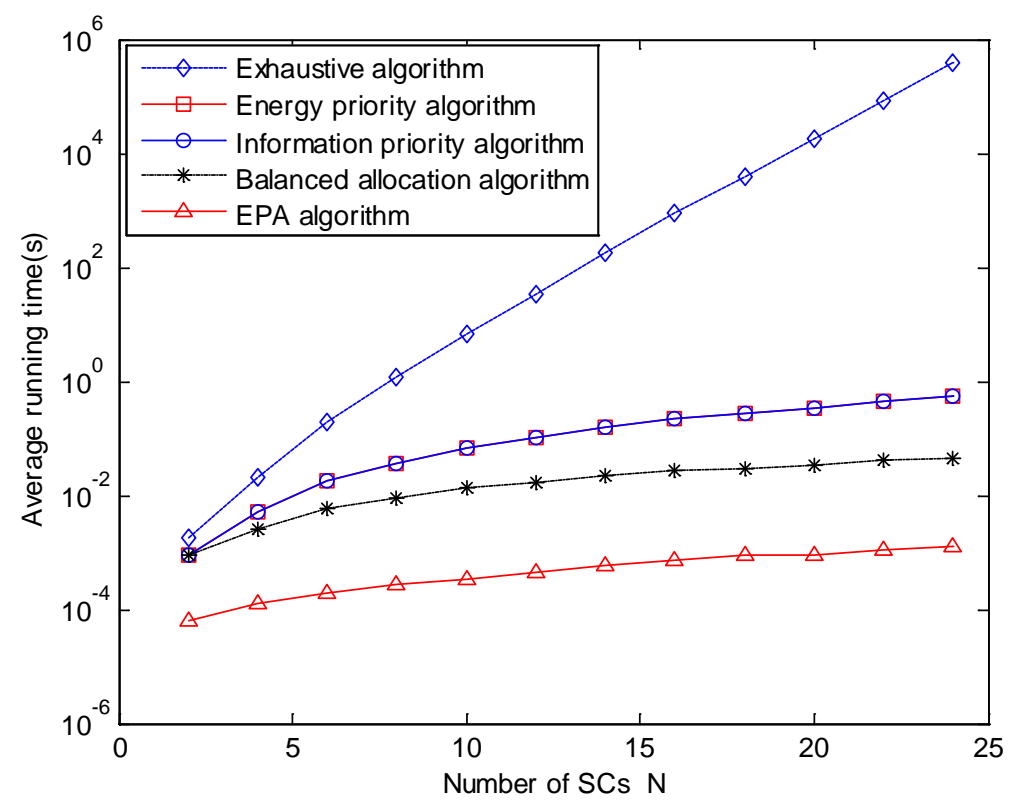

Fig. 8. The average running time comparison of different algorithms under different $N$, where

$$
Q=26 \mathrm{dBm}, P^{\max }=17 \mathrm{dBm}
$$

\section{Conclusions}

In this paper, an OFDM relay network based on SWIPT is studied. We propose a resource allocation algorithm by maximizing the system throughput, and the algorithm can flexibly adjust the number of the SCs used to transfer energy according to the channel condition. Firstly, with the given SC allocation of the source, we give and prove the optimal propositions of the formulated problem. Then, the formulated problem could be decomposed into two separate throughput maximization sub-problems by setting the total power to transfer energy from the source to the relay. The results reveal that the energy priority algorithm can achieve approximate performance with the exhaustive scheme, and is superior to the other algorithms.

\section{References}

[1] L. R. Varshney, "Transporting information and energy simultaneously," in Proc. of 2008 IEEE International Symposium on Information Theory, pp. 1612-1616, July 6-11, 2008. Article (CrossRef Link)

[2] X. Zhou, R. Zhang and C. K. Ho, "Wireless Information and Power Transfer: Architecture 
Design and Rate-Energy Tradeoff,” IEEE Transactions on Communications, vol. 61, no. 11, pp. 4754-4767, Nov. 2013. Article (CrossRef Link)

[3] X. Lu, P. Wang, D. Niyato, D. I. Kim and Z. Han, "Wireless Networks With RF Energy Harvesting: A Contemporary Survey,” IEEE Communications Surveys and Tutorials, vol. 17, no. 2, pp. 757-789, 2015. Article (CrossRef Link)

[4] M. L. Ku, W. Li, Y. Chen and K. J. R. Liu, "Advances in Energy Harvesting Communications: Past, Present, and Future Challenges,” IEEE Communications Surveys and Tutorials, vol. 18, no. 2, pp. 1384-1412, 2016. Article (CrossRef Link)

[5] N. U. Hasan, W. Ejaz, N. Ejaz, H. S. Kim, A. Anpalagan and M. Jo, "Network Selection and Channel Allocation for Spectrum Sharing in 5G Heterogeneous Networks," IEEE Access, vol. 4, pp. 980-992, Mar. 2016. Article (CrossRef Link)

[6] M. Dai, S. Zhang, B. Chen, X. Lin and H. Wang, "A Refined Convergence Condition for Iterative Waterfilling Algorithm,” IEEE Communications Letters, vol. 18, no. 2, pp. 269-272, Feb. 2014. Article (CrossRef Link)

[7] B. B. Bai, W. Chen, K. B. Letaief and Z. Cao, "A Unified Matching Framework for Multi-Flow Decode-and-Forward Cooperative Networks," IEEE Journal on Selected Areas in Communications, vol. 30, no. 2, pp. 397-406, Feb. 2012. Article (CrossRef Link)

[8] O. Ozel, K. Tutuncuoglu, J. Yang, S. Ulukus and A. Yener, "Transmission with Energy Harvesting Nodes in Fading Wireless Channels: Optimal Policies,” IEEE Journal on Selected Areas in Communications, vol. 29, no. 8, pp. 1732-1743, Sep. 2011. Article (CrossRef Link)

[9] B. Varan and A. Yener, "Delay Constrained Energy Harvesting Networks with Limited Energy and Data Storage," IEEE Journal on Selected Areas in Communications, vol. 34, no. 5, pp. 1550-1564, May 2016. Article (CrossRef Link)

[10] H. Gao, W. Ejaz and M. Jo, "Cooperative Wireless Energy Harvesting and Spectrum Sharing in 5G Networks,” IEEE Access, vol. 4, pp. 3647-3658, July 2016. Article (CrossRef Link)

[11] M. C. Ju, K. M. Kang, K. S. Hwang and C. Jeong, "Maximum Transmission Rate of PSR/TSR Protocols in Wireless Energy Harvesting DF-Based Relay Networks," IEEE Journal on Selected Areas in Communications, vol. 33, no. 12, pp. 2701-2717, Dec. 2015. Article (CrossRef Link)

[12] B. S. Xu, Y. Zhu and R. Zhang, "Optimized Power Allocation for Interference Channel with SWIPT,” IEEE Wireless Communications Letters, vol. 5, no. 2, pp. 220-223, Apr. 2016. Article (CrossRef Link)

[13] C. J. Zhong, H. A. Suraweera, G. Zheng, I. Krikidis and Z. Y. Zhang, "Wireless Information and Power Transfer With Full Duplex Relaying,” IEEE Transactions on Communications, vol. 62, no. 10, pp. 3447-3461, Oct. 2014. Article (CrossRef Link)

[14] R. Feng, M. Dai and H. Wang, "Distributed Beamforming in MISO SWIPT System,” IEEE Transactions on Vehicular Technology, vol. 66, no. 6, pp. 5440-5445, June 2017. Article (CrossRef Link)

[15] C. Hsu, H. Su and P. Lin, "Joint Subcarrier Pairing and Power Allocation for OFDM Transmission With Decode-and-Forward Relaying," IEEE Transactions on Signal Processing, vol. 59, no. 1, pp. 399-414, Jan. 2011. Article (CrossRef Link)

[16] X. Li, Q. Zhang, G. Zhang and J. Qin, "Joint Power Allocation and Subcarrier-Relay Assignment for OFDM-Based Decode-and-Forward Relay Networks," IEEE Communications Letters, vol. 17, no. 5, pp. 872-875, May 2013. Article (CrossRef Link)

[17] A. Attarkashani and W. Hamouda, "Joint Power Allocation and Subcarrier-Relay Assignment for OFDM-Based Decode-and-Forward Relay Networks,” IEEE Communications Letters, vol. 20, no. 11, pp. 2312-2315, Nov. 2016. Article (CrossRef Link) 
[18] K. B. Huang and E. Larsson, "Simultaneous Information and Power Transfer for Broadband Wireless Systems,” IEEE Transactions on Signal Processing, vol. 61, no. 23, pp. 5972-5986, Dec. 2013. Article (CrossRef Link)

[19] D. W. K. Ng, E. S. Lo and R. Schober, "Wireless Information and Power Transfer: Energy Efficiency Optimization in OFDMA Systems," IEEE Transactions on Wireless Communications, vol. 12, no. 12, pp. 6352-6370, Dec. 2013. Article (CrossRef Link)

[20] M. Zhang and Y. Liu, "Energy Harvesting for Physical-Layer Security in OFDMA Networks," IEEE Transactions on Information Forensics and Security, vol. 11, no. 1, pp. 154-162, Jan. 2016. Article (CrossRef Link)

[21] X. Zhou, R. Zhang and C. K. Ho, "Wireless Information and Power Transfer in Multiuser OFDM Systems,” IEEE Transactions on Wireless Communications, vol. 13, no. 4, pp. 2282-2294, Apr. 2014. Article (CrossRef Link)

[22] W. Lu, Y. Gong, J. Wu, H. Peng and J. Hua, "Simultaneous Wireless Information and Power Transfer Based on Joint Subcarrier and Power Allocation in OFDM Systems," IEEE Access, vol. 5, pp. 2763-2770, Feb. 2017. Article (CrossRef Link)

[23] X. Zhou, C. K. Ho and R. Zhang, "Wireless Power Meets Energy Harvesting: A Joint Energy Allocation Approach in OFDM-Based System," IEEE Transactions on Wireless Communications, vol. 15, no. 5, pp. 3481-3491, May 2016. Article (CrossRef Link)

[24] L. He, G. Zhang, M. Cui, H. Tong, Y. Liu, G. Huang and F. Lin, "Joint Time Switching and Power Allocation in Large-Scale MISO OFDM Systems with Wireless Powered Relay," in Proc. of 2016 IEEE International Conference on Consumer Electronics-China (ICCE-China), pp. 1-5, July 17-21, 2016. Article (CrossRef Link)

[25] K. Xiong, P. Fan, C. Zhang and K. B. Letaief, "Wireless Information and Energy Transfer for Two-Hop Non-Regenerative MIMO-OFDM Relay Networks," IEEE Journal on Selected Areas in Communications, vol. 33, no. 8, pp. 1595-1611, Aug. 2015. Article (CrossRef Link)

[26] Y. Liu and X. D. Wang, "Information and Energy Cooperation in OFDM Relaying: Protocols and Optimization,” IEEE Transactions on Vehicular Technology, vol. 65, no. 7, pp. 5088-5098, Jul. 2016. Article (CrossRef Link)

[27] Y. Zeng and R. Zhang, "Optimized training design for wireless energy transfer," IEEE Transactions on Communications, vol. 63, no. 2, pp. 536-550, Feb. 2015. Article (CrossRef Link)

[28] I. Krikidis, S. Timotheou, S. Nikolaou, G. Zheng, D. W. K. Ng and R. Schober, "Simultaneous wireless information and power transfer in modern communication systems," IEEE Communications Magazine, vol. 52, no. 11, pp. 104-110, Nov. 2014. Article (CrossRef Link)

[29] S. Bi, C. K. Ho and R. Zhang, "Wireless powered communication: opportunities and challenges,” IEEE Communications Magazine, vol. 53, no. 4, pp. 117-125, Apr. 2015. Article (CrossRef Link)

[30] M. Konstantinos, A. Adamis and P. Constantinou, "Receiver architectures for OFDMA systems with subband carrier allocation,"' in Proc. of 14th European Wireless Conference, pp. 1-7, June 22-25, 2008. Article (CrossRef Link)

[31] S. Boyd and L. Vandenberghe, Convex Optimization. Cambridge University Press, New York, NY, USA, 2004. Article (CrossRef Link)

[32] A. J. Goldsmith, Wireless Communications. Cambridge University Press, New York, NY, USA, 2005. Article (CrossRef Link) 


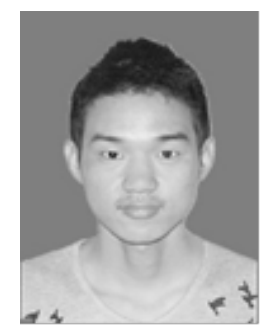

Zhenwei Xie was born in Henan Province, China, in 1991. He received the B.E. degree in Communication Engineering from Jiangnan University, Wuxi, in 2015. He is now pursuing master's degree in the department of Communication and Information Engineering, Nanjing University of Posts and Telecommunications, Jiangsu, China. He researches on the area of resource management, relay selection, energy harvesting and subcarrier paring in cooperative networks.

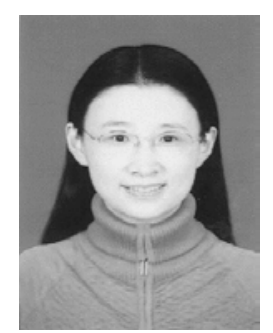

Qi Zhu (corresponding author) was born in Suzhou, Jiangsu, China, in 1965. She received the M.S. degree in radio engineering from Nanjing University of Posts and Telecommunications in 1989. Now she is a professor in the Department of Telecommunication and Information Engineering, Nanjing University of Posts and Telecommunications, Jiangsu, China. Her research interests focus on technology of next generation communication, broadband wireless access, OFDM, channel and source coding, dynamic allocation of radio resources.

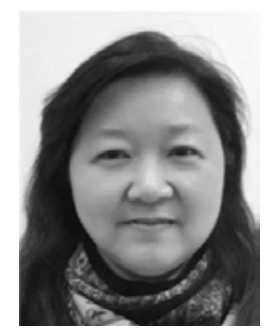

Su Zhao received the M.S. degree in communication and information system from Beijing University of Posts and Telecommunications in 2005. Now she is an associate professor in the Department of Telecommunication and Information Engineering, Nanjing University of Posts and Telecommunications, Jiangsu, China. Her research interests focus on MIMO, radio resource dynamic allocation, modulation and demodulation and cognitive radio networks. 